\title{
Guía metodológica para la selección de proveedores DBaaS en Pymes
}

\author{
Methodological Guide for the Selection of DBaaS Providers in SMEs
}

DBaaS Guia de Metodologia de Selecção de Fornecedores para PME

\author{
Fabián Leonardo Vargas-Corredor ${ }^{1}$ \\ Jorge Enrique Quevedo-Reyes ${ }^{2}$ \\ Juan Sebastián González-Sanabria ${ }^{3}$ \\ Marco Javier Suárez-Barón ${ }^{4}$
}

Recibido: marzo 2020

Aceptado: agosto 2020

Para citar este artículo: Vargas-Corredor, F. L., Quevedo-Reyes, J. E., González-Sanabria, J. S., Suárez-Barón, M. J. (2020). Guía Metodológica para la selección de proveedores DBaaS en Pymes. Revista Científica, 39(3), 369-377. https://doi.org/10.14483/23448350.16938

\section{Resumen}

La importancia que han adquirido las bases de datos como servicio (DBaaS) y la relación que tienen los proveedores directamente en la gestión de la información cuando se ofrece un servicio en la nube son factores clave en el manejo de la información. El impacto negativo en los recursos monetarios, desempeño y seguridad de la información ha inspirado un análisis en la adquisición de servicios en la nube en el que se ha buscado maneras para mitigar errores en la adopción de DBaaS. El presente trabajo pretende plantear el desarrollo de una guía metodológica que fortalezca los procesos de adquisición de DBaaS en pequeñas y medianas empresas (Pymes), logrando minimizar su impacto en la pérdida o inutilización de recursos monetarios, infraestructura y seguridad. Para lo anterior se realizó una revisión documental incluyendo documentos publicados entre 2008 a 2018, periodo en el que países con niveles de desempeño altos en investigación sobre computación en la nube y servicios en la gestión de las bases de datos publicaron de manera significativa. La creación de una guía metodológica en la selección de proveedores de DBaaS en Pymes establece métodos, caminos y estrategias sobresalientes al momento de adquirir un servicio DBaaS. La prioridad es conocer en detalle cómo se desarrollan y se gestionan los acuerdos de nivel de servicio orientados a la adopción de servicios Cloud.

Palabras clave: respaldo de la información, DBaaS, CSP, computación en la nube, seguridad en la nube, migración de proveedores de servicios.

\section{Abstract}

The importance that has acquired the trend of database-as-a-service (DBaaS) and the relationship that providers have directly in the management of information when offering a service in the cloud, are key

1. Universidad Pedagógica y Tecnológica de Colombia. Tunja, Boyacá. Colombia. fabianleonardo.vargas@uptc.edu.co

2. Universidad Pedagógica y Tecnológica de Colombia. Tunja, Boyacá. Colombia. jorge.quevedo@uptc.edu.co

3. Universidad Pedagógica y Tecnológica de Colombia. Tunja, Boyacá. Colombia juansebastian.gonzalez@uptc.edu.co

4. Universidad Pedagógica y Tecnológica de Colombia. Tunja, Boyacá. Colombia marco.suarez@uptc.edu.co 
factors in the management of information. The negative impact on monetary resources, performance and information security has inspired an analysis on cloud service acquisition in order to mitigate errors in DBaaS adoption. The present work intends to propose the development of a methodological guide that strengthens the DBaaS acquisition processes in SMEs, managing to minimize its impact on the loss or disuse of monetary resources, infrastructure, and security. For this purpose, a document review is carried out, including documents from 2008 to 2018, a period in which countries with high performance levels in research on cloud computing and database management services published significantly. The creation of a methodological guide in the selection of DBaaS providers in SMEs, establishes methods, paths and outstanding strategies when acquiring a DBaaS service. The priority is to know in detail how the service level agreements oriented to the adoption of Cloud Services are developed and managed.

Keywords: information backup, dbaas, csp, cloud computing, cloud security, service provider migration.

\section{Resumo}

A importância das Bases de Dados como Serviço (DBaaS) e a relação que os fornecedores têm directamente na gestão da informação quando oferecem um serviço na nuvem são factores chave na gestão da informação. O impacto negativo nos recursos monetários, desempenho e segurança da informação inspirou uma análise da aquisição de serviços na nuvem, a fim de mitigar erros na adopção de DBaaS. Este trabalho visa desenvolver um guia metodológico que reforce os processos de aquisição de DBaaS nas PMEs, conseguindo minimizar o seu impacto na perda ou desuso de recursos monetários, infra-estruturas e segurança. Para este fim, está a ser realizada uma revisão documental, incluindo documentos de 2008 a 2018, período em que os países com elevados níveis de desempenho na investigação sobre computação em nuvem e serviços de gestão de bases de dados publicaram significativamente. A criação de um guia metodológico na selecção de fornecedores de DBaaS nas PMEs, estabelece métodos, caminhos e estratégias notáveis ao adquirir um serviço DBaaS. A prioridade é saber em detalhe como são desenvolvidos e geridos os acordos de nível de serviço orientados para a adopção de Serviços em Nuvem.

Palavras-chaves: backup de informação, dbaas, csp, cloud computing, segurança na nuvem, migração de prestadores de serviços.

\section{Introducción}

El uso de la tecnología y la información se ha convertido en una ventaja competitiva en los negocios, puesto que se puede generar un beneficio económico en la reducción de costos y uso efectivo de los recursos (ya sea que se trate de factores en cuanto al software, hardware, infraestructura o gestión) (Prieto y Ariaz, 2014). La clave del éxito para algunas economías se ve reflejada en la innovación de sus procesos y productos tecnológicos que tienen como finalidad competir en el mercado (Senarathna, 2016). Actualmente, las pequeñas y medianas empresas (Pymes) surgen y crecen de manera acelerada, por lo que requieren una atención constante al momento de adquirir los servicios que ofrecen los proveedores de servicios cloud (CSP, por sus siglas en inglés) (Maqueira y Bruque, 2012).

Debido al impacto en costos y eficacia presentada en las organizaciones que han adoptado las bases de datos como servicio (DBaaS), se propone una provisión para la gestión de la computación en la nube en los próximos años en la que se consideren servicios que puedan ser consumidos a través de internet de forma inmediata y segura (Alzoubaidi, 2016). Asimismo, las Pymes han estructurado su información de modo que puedan ser dirigidas a este tipo de servicios (Zuberoa et al., 2017). Sin embargo, es evidente que el incremento $y$ la competitividad en el mercado han hecho que las organizaciones no puedan adquirir los paquetes integrados que ofrecen los CSP, como en el caso de que algunos clientes necesiten solamente un servicio que satisfaga sus necesidades en la gestión tecnológica (Matthew et al., 2014).

Por lo anterior, es necesario reflexionar sobre el impacto que genera la creación de una guía 
metodológica en la selección de proveedores de DBaaS para Pymes, con el fin de establecer métodos y caminos que comprendan factores pertinentes al momento de adquirir este servicio (Hentschel et al., 2018). La tendencia que han generado los DBaaS se ha visto de forma prometedora en función del recurso que asignan los proveedores de bases de datos, pues su visión en conjunto es integrar los aspectos relevantes que contienen los catálogos de SLA (Gunarathne et al., 2011). El coste, la disponibilidad, la integración y la independencia de servicios no son los únicos aspectos que impulsan a las empresas a adoptar DBaaS, también existen factores como autoservicio por demanda, acceso ubicuo a la red, fondo común de recursos, rápida elasticidad y servicio medido (Wong et al., 2013). Para otros clientes, según la investigación de Microsoft y la Universidad de Utah (Du y Ramamurthy, 2017), la prioridad es conocer en detalle cómo se desarrollan y se gestionan los SLA. En esencia, se evalúan tres criterios para identificar el manejo de excepciones: revisión del catálogo de negocios, catálogo de servicio mismo y el catálogo técnico (Clouse, 2016).

\section{Antecedentes de investigación}

La computación en la nube (cloud computing) se ha convertido en un modelo que permite el acceso adecuado a una red compartida de recursos informáticos; en esta pueden ser configurables, por ejemplo, servidores de almacenamiento, aplicaciones y servicios. Además, se pueden aprovisionar y liberar rápidamente con un mínimo esfuerzo de gestión o interacción (Alzoubaidi, 2016). Desde la perspectiva del Departamento de Ciencias de la Información y Tecnología de la Universidad de Penn State (Aiken et al., 2015) se define como un modelo de implementación de hardware y software en el cual el dispositivo de software se aloja en hardware de alto rendimiento y se proporciona como un servicio a los usuarios a través de internet. La eliminación de la necesidad de instalar, configurar y ejecutar aplicaciones en los propios servidores y locales de los usuarios contribuye significativamente a reducir la carga del mantenimiento del software y el funcionamiento continuo (Cuzzocre et al., 2016). Al mismo tiempo, los recursos de TI deben ser pagados a través de una suscripción en lugar de una licencia; lo cual significa que las empresas no necesitan adquirir e instalar los recursos de alto costo en sus sitios (Alzoubaidi, 2016).

En otras palabras, los DBaaS son tipos de servicios recientes que se están investigando en la comunidad de computación en la nube (Cuzzocre et al., 2016). Desde el punto de vista funcional, el principal objetivo de DBaaS es superar las limitaciones de los enfoques de vanguardia en tecnologías de datos, según se almacenan y se acceden desde repositorios cuya ubicación es conocida y relevante para compartir y procesar información.

\section{Metodología}

Se realizó una revisión documental tal como lo propone Serrano (2014), quien define cinco fases para elaborar un estado de la cuestión en un área de investigación. La revisión incluyó documentos desde el año 2008 hasta el 2018; pues en este periodo se publicó la mayoría de artículos de los países con niveles de desempeño altos en investigación sobre computación en la nube y los servicios que aportan a la gestión de las bases de datos (Zhang et al., 2010).

La primera fase de preparación tiene por objetivo general identificar el grado de discernimiento que poseen las Pymes en la adopción de CSP, especialmente DBaaS. En la segunda fase se aborda un enfoque descriptivo para definir el tema central. En resumen, este artículo se enfoca en la adopción de servicios DBaaS en Pymes y se estudiarán los autores representativos. Por otra parte, se usan fichas descriptivas que corresponden a la exploración de estudios relacionados con el tema principal.

En la tercera fase de interpretación por núcleos se exploran las fichas bibliográficas, así como se interpretan los datos concernientes a las 
características relevantes determinadas por los proveedores de DBaaS. Es decir, se hizo un análisis de información en las fichas bibliográficas a través de comparativas de datos coincidentes. En la cuarta fase, sobre construcción teórica global, se identifican los posibles vacíos, oportunidades y campos de aplicación correspondientes a los servicios DBaaS ofrecidos por los CSP, particularmente en el sector de las pymes. La quinta fase, de expansión y publicación, se plantea como inicio de una próxima publicación sobre posibles modelos que representan un camino acertado en la adopción de DBaaS.

El alcance de este trabajo es descriptivo y se ha realizado a partir de un ejercicio de revisión de literatura sobre DBaaS, cuyo fin es establecer los factores que interfieren en la adopción de CSP en Pymes; se estudia desde un enfoque cualitativo y cuantitativo considerando el nivel de satisfacción de los clientes. Se usa una ventana de investigación desde el año 2008 hasta el 2018, donde los criterios de búsqueda definen los vacíos sobre una guía que permite el estudio profundo de estos elementos.

\section{Resultados}

\section{Aplicaciones de guías metodológicas para resolver aspectos que interfieren en la adopción de DBaaS}

En la actualidad diversas organizaciones han migrado el almacenamiento de sus datos desde servidores físicos hacia la nube, por la evidente disminución en costos que esto representa. Por su parte, los DBaaS han tenido gran auge en la nueva era tecnológica. Sin duda estos enfocan su objetivo en la gestión de los datos, de modo que las funciones ofrecidas por los CSP prometen soluciones a necesidades relacionadas con dos aspectos importantes: la reducción de costos y el rendimiento del sistema; esto con el fin de apuntar los esfuerzos al beneficio de la organización (Nishad et al., 2016).
Se consideró que en la realización de la guía metodológica para la selección de proveedores DBaaS las Pymes deben estimar qué tipo de información se utiliza. Es decir, qué tan sensible puede ser a los ciberataques y la divulgación accidental pues, en colaboración con los aportes ofrecidos por la revista en su apartado predicción de $\mathrm{TI}$ (Gartner, 2016), se evidencia que el $18,1 \%$ de todos los documentos cargados en servicios de colaboración y uso compartido de archivos basados en la nube contienen información confidencial.

En el Departamento de Ciencias de la Computación e Ingeniería de la Computación de la Universidad de Jamia Millia Islamia en Nueva Delhi (India) (Malhotra et al., 2015) se considera que los lugares donde se gestionan los datos determinan el respaldo de las Pymes, de forma que garantizan la tranquilidad en la administración de su información (Cuzzocre et al., 2016). En la figura 1 se destacan elementos en los que un servicio DBaaS está comprometido a diario, estimando que el activo más importante de cualquier organización es la información (Marston et al., 2011).
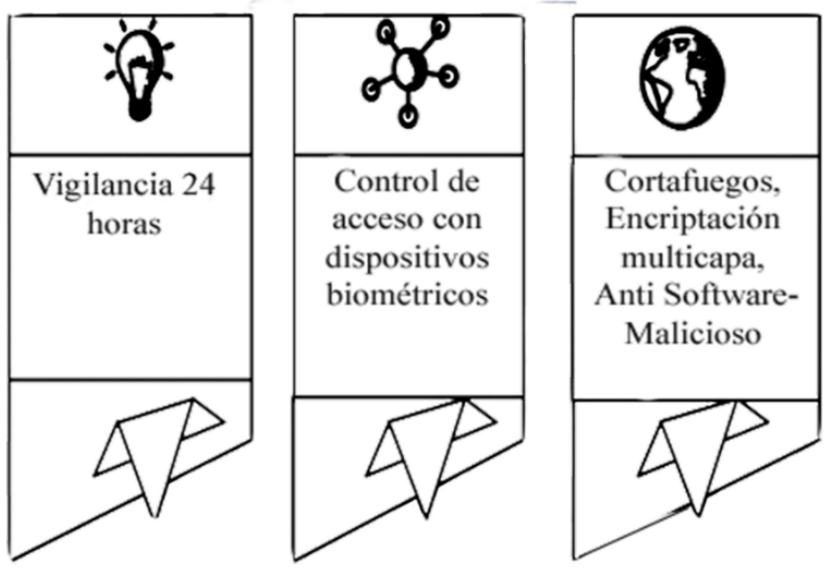

Figura 1. Elementos para la gestión de la seguridad. Fuente: elaboración propia de los autores.

Para proporcionar una visión más cercana de las características que presenta una guía metodológica, en relación con la selección de proveedores de servicios de DBaaS, se ha dividido en una comparativa de seis aspectos principales: conceptos 
básicos de la plataforma, calidad de servicio, costo del servicio, posicionamiento del proveedor en el mercado, industrias del mercado que considerablemente hacen uso de implementaciones DBaaS $y$, finalmente, tendencias en el mercado e impacto a corto plazo en DBaaS (Henderson, 2016).

En relación con lo anterior, los componentes necesarios que debe incluir la guía para la adopción de DBaaS son:

- Disponibilidad: uno de los factores relevantes en la selección del CSP es la calidad del servicio con respecto a los tiempos de atención y resolución de problemas que se presentan en la plataforma.

- Seguridad: al momento de resguardar la información es clave saber qué métodos se usan y en qué lugar se realiza, pues los protocolos de administración y políticas de respaldo deben ser de alto nivel (Mehak et al., 2014). Ahora bien, es vital revisar el orden en que pueden amenazar a la organización, pues este tipo de acciones se ejecuta de la siguiente manera: persona enterada, cuentas comprometidas, privilegios de usuarios y fugas de información.

- Capacidad: indiscutiblemente la tecnología que usan los CSP hace la diferencia para que proporcionen un servicio de acuerdo con la solicitud del cliente. Es decir, si requiere que sea almacenada su información en un disco de estado sólido o un disco duro y cuánta capacidad es necesaria para que funcione de forma eficiente el sistema.

- Elasticidad rápida: las arquitecturas DBaaS deben apoyar la elasticidad del servicio granular, una propiedad de múltiple seguridad que define el acceso usando una amplia gama de dispositivos y mecanismos no propietarios. Además, debe ser analizada desde el punto de vista de la gestión automatizada de recursos y la planificación integrada de la capacidad (Tomiyama et al., 2012).

- Rendimiento: el desempeño del servicio debe estar ligado a los requisitos de los dispositivos, de modo que la calidad y el rendimiento superen aspectos como capacidad de procesamiento, modelo de almacenamiento e integración y velocidad de respuesta.

- Carga de trabajo: en ocasiones suele suceder que los dispositivos en los cuales corren los servicios presenten cierto tipo de fallas; por lo que las cargas de trabajo deben distribuirse de manera eficiente.

- Soporte: los CSP cuentan con un equipo de soporte en la plataforma tecnológica del DBaaS, es por esto por lo que la mayoría de los proveedores incluye como parte de la facturación el valor por el soporte. Los proveedores (Casola et al., 2018) manejan un esquema de soporte con tiempos de respuesta no mayores a un día (en horario cinco días por nueve horas); en algunos casos en un tiempo récord, casi inmediato.

- Costos: cada proveedor de DBaaS ofrece esquemas de costo particulares. Para algunos CSP su modelo de negocio es entregar una solución establecida con características de hardware preconfiguradas por un costo de suscripción fijo mensual. En el caso de cambiar a un esquema diferente simplemente se modifica su configuración de acuerdo con sus nuevas necesidades.

- SLA: el acuerdo de nivel de servicio proporciona una colección de estándares cuantificables y mensurables que el cliente percibe necesarios para el uso efectivo de los servicios del CSP (Hoehl, 2016). De ahí que los estándares sean un componente clave dentro del contrato de servicios, pues con ello se asegura el cumplimiento de los objetivos comerciales. En el contexto de acuerdos de servicio de los CSP, se puede llegar a penalizaciones por incumplimiento del servicio.

\section{Guía metodológica como marco general en la adopción de servicios en la nube}

Con la creación de una guía metodológica para la adecuada selección de DBaaS se consigue una idea clara en función de la correcta administración de dichos servicios. Por la razón anterior, se entiende 
como un marco que permite la transparencia y visibilidad en aspectos tales como: servicio completo y controlado, gestión unificada, seguridad en Pymes, operaciones en la nube, asignación de recursos, gestión de roles, servicio de consultoría en la nube, elasticidad, SLA, costes totales, atención al cliente, políticas, zonas de operación, automatización de la nube, gestión de riesgos, agilidad empresarial y operaciones de TI simplificadas. Sin embargo, las estrategias de implementación de un servicio DBaaS deben estar vinculadas a la mejora continua del negocio en las Pymes, ya que cuando se decide incluir este servicio se debe analizar el soporte de TI necesario y qué tan viable es la integración entre los productos.

La guía sugiere comprender la estrategia de negocio en relación con TI, y sus pasos determinan las fases que se analizan al momento de adoptar un DBaaS. A continuación, se describe cada una de ellas.

- Fase revisión preliminar: comprende la estrategia de negocio en relación con los requisitos de TI. Naturalmente, se evalúa la posibilidad de implementación. En esta fase se lleva a cabo el estudio de oportunidades, desafíos y las posibles opciones para poner en marcha servicios en la nube.

- Fase estudio de necesidades: en este ítem se analizan los vacíos y cómo se podrían generar soluciones a estas dificultades; también se clasifican sistemas de información y se estudia la posible integración con los sistemas actuales, puesto que el objetivo es reducir costos y tiempo. Finalizado el resultado se determina el nivel de capacidad, velocidad, seguridad y disponibilidad del servicio, sin dejar atrás la percepción de barreras y posibles riesgos antes, durante y después del cambio; es aquí donde se deben identificar los procedimientos con los que se involucra este servicio y cómo se podría prosperar. En esta sección se ofrece una perspectiva sobre las necesidades más relevantes en las organizaciones, especialmente sobre características de desempeño.
- Fase as to be: además de realizar el estudio de los vacíos, se proyecta cómo se desearía que funcionara el sistema con la implementación de un DBaaS, de modo que se piense en la operación, la gestión, la confidencialidad y los recursos empleados. Además de identificar las aplicaciones que podrían funcionar, también es pertinente hacer el estudio de satisfacción y sencillez. De hecho, es recomendable seleccionar aplicaciones que sean simples de migrar e independientes del sistema principal. Por este motivo es aconsejable adquirir un porcentaje mínimo de aplicaciones con el fin de aprender de los procesos de productividad, agilidad y eficiencia en la nube, para posteriormente aplicar la experiencia aprendida en sistemas más complejos. En esta sección se especifican los niveles de adopción de DBaaS, comprendidos de la siguiente forma: nivel de adopción mínimo: 30 \% de la implementación; nivel de adopción intermedio: se aproxima al $50 \%$ de la adopción; nivel de adopción final: supera el $50 \%$ de la implementación.

- Fase examen de proveedores: en primera instancia es importante revisar las políticas y acuerdos en relación con casos de éxito sobre organizaciones que usan su servicio, sin dejar de lado el costo que representa adoptar el DBaaS. Considérese que cada CSP ofrece diferentes funciones, dentro de ellas la tarea de migración y establecimiento del mapa de ruta. Las fases de estudio de necesidades y examen de proveedores están ligadas, ya que se comprometen a estudiar los estándares de adopción, portabilidad y metodologías para migrar aplicaciones. Por otra parte, se sugiere revisar los SLA que ofrece cada proveedor y crear así un listado de los servicios que se verían afectados y cómo se podría reestructurar de acuerdo con la arquitectura planteada en la fase as to be. Un aspecto importante en esta fase es revisar el modelo de seguridad 
que ofrecen dichos CSP; de hecho, la infraestructura, la capacidad y el cumplimiento de las normas hacen posible la correcta operación y transparencia en el despliegue de sus aplicaciones.

- Fase factores de operación: considerando que cada país interpreta de manera distinta sus aspectos legales, en esta última fase se estudian los factores de operación tales como fácil separación o migración de la información, así como también normas que regulen la protección y privacidad de datos en los servicios de computación en la nube. Otra condición para identificar la calidad en la prestación del servicio es revisar costos, licencias, contratos de confidencialidad con terceros y garantías en caso de ocurrir un desastre.

En la figura 2 se proponen las fases en que la guía metodológica ordena el proceso de adopción de DBaaS; en esta se considera como parte fundamental la opinión del cliente. Sin embargo, se describe como un ciclo iterativo donde pueda interactuar entre fases, de modo que se pueda hacer retroalimentación de cada fase.

\section{Discusión y conclusiones}

El análisis sobre la selección de proveedores de bases de datos como servicio determinó que las Pymes experimentan cambios en el uso de la información rápidamente, por tal razón es necesario determinar factores de adopción en DBaaS que contribuyan al desarrollo de esta. Nosotros observamos que las Pymes actualmente no disponen de una guía metodológica que integre todos los aspectos importantes al momento de escoger un CSP; algunas de ellas enfocan su objetivo en diferentes necesidades, destacándose entre otras, costos, riesgos, seguridad y desempeño.

Además, se percibieron las falsas expectativas del modelo de mercado actual que se ofrece en el área de DBaaS. Por tal razón los servicios no se usan o probablemente no satisfacen las necesidades de los clientes. Así que, teniendo en cuenta lo anterior, nosotros proponemos los criterios que debería tener una guía metodología, incluyendo los ajustes que se adapten a la normativa que rige en el país, sin dejar de lado los riesgos económicos que puedan llegar a impactar o incluso su nivel de demanda de servicios en las Pymes.

\section{FASES DE LA GUÍA METODOLÓGICA}

\section{FASE PRELIMINAR \\ Protección \\ Privacidad \\ Confidencialidad \\ ESTUDIO DE \\ NECESIDADES \\ Estado actual y posibles brechas}

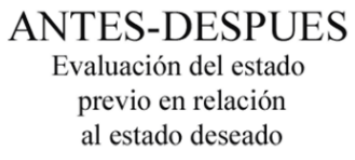

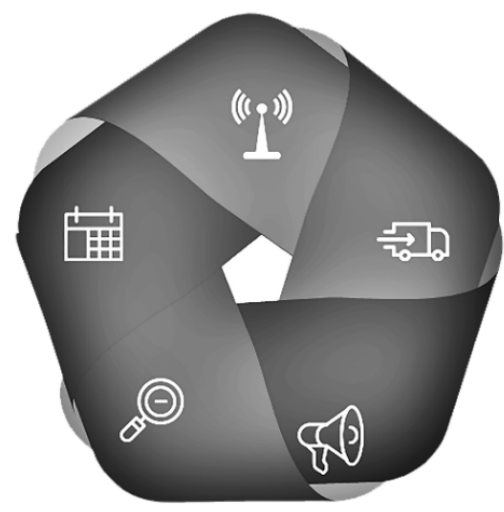

EXAMEN DE PROVEEDORES

Análisis de las políticas

y características ofrecidas por cada proveedor

FACTORES DE
OPERACIÓN
Entendimiento del negocio
Estrategia en relación a TI

Figura 2. Fases de la guía.

Fuente: elaboración propia de los autores. 


\section{Referencias}

Aiken, W., Ryoo, J., Kim, H. (2015). KaaSP: keying as a service provider for small and medium enterprises using untrusted cloud services. Paper presented at the Proceedings of the 9th International Conference on Ubiquitous Information Management and Communication, Bali, Indonesia. https://doi.org/10.1145/2701126.2701206

Alzoubaidi, A. R. (2016). Private-Cloud Computing Services for an Interactive Multi-Campus University illM, 10(4). https://doi.org/10.3991/ijim.v10i4.5931

Casola, V., Benedictis, A. D., Modic, J., Rak, M., Villano, U. (2018). Per-service security SLAs for cloud security management: Model and implementation. International Journal of Grid and Utility Computing, 9(2), 128-138. https://doi.org/10.1504/IJGUC.2018.091719

Clouse, B. (2016). Service Catalogs: Defining Standardized Database Services. In O. Corporation (Ed.), World Headquarters (vol. 1.6, pp. 2-43). Redwood City, CA 94065 USA.

Cuzzocre, A., Mastroianni, C., Grasso, G. M. (2016). Private Databases on the Cloud: Models, Issues and Research Perspectives. IEEE International Conference on Big Data (Big Data). https://doi. org/10.1109/BigData.2016.7841032

Du, M., Ramamurthy, R. (2017). Towards verifiable metering for database as a service providers. Symposium on Cloud Computing, Santa Clara, California. https://doi.org/10.1145/3127479.3134349

Gartner (2016). Predicciones de la agenda 2019 de los $\mathrm{CIO}$ en todo el mundo. https://datta.com.ec/articulo/predicciones-dela-agenda-2019-de-los-cio-en-todo-el-mundo

Gunarathne, T., Wu, T. L., Jong, Y. C., Bae, S. H., Qiu, J. (2011). Cloud computing paradigms for pleasingly parallel biomedical applications. Concurrency and Computation: Practice and Experience, 23(17), 2338-2354. https://doi.org/10.1002/cpe.1780
Henderson, N. (2016). Three Cloud Trends to Watch Through 2020. https://cutt.ly/QfvHUUR

Hentschel, R., Leyh, C., Petznick, A. (2018). Current cloud challenges in Germany: the perspective of cloud service providers. Journal of Cloud Computing, 7(1). https://doi.org/10.1186/ s13677-018-0107-6

Hoehl, M. (2016). Proposal for standard Cloud Computing Security SLAs-Key Metrics for Safeguarding Confidential Data in the Cloud. https://cutt.ly/WfvHSIk

Malhotra, S., Doja, M., Alam, B., Alam, M. (2015). Data integration of cloud-based and relational databases. International Conference on Soft Computing Techniques and Implementations (ICSCTI). https://doi.org/10.1109/ICSCTI.2015.7489542

Maqueira, J., Bruque, S. (2012). Agentes impulsores de la adopción de Cloud Computing en las empresas. ¿Quién mueve la nube? Universia Business Review, 3(35).

Marston, S., Li, Z., Bandyopadhyay, S., Zhang, J., Ghalsasi, A. (2011). Cloud computing-The business perspective. Decision Support Systems, 51(1), 176-189. https://doi.org/10.1016/j.dss.2010.12.006

Matthew, O., Dudley, C., Moreton, R. (2014). A review of multi-tenant database and factors that influence its adoption. Ukais, 22.

Mehak, F., Masood, R., Ghazi, Y., Shibli, A., Khan, S. (2014). Security Aspects of Database-as-a-Service (DBaaS) in Cloud Computing. https://doi. org/10.1007/978-3-319-10530-7 13

Nishad, L. S., Akriti, Paliwal, J., Pandey, R., Beniwal, S., Kumar, S. (2016). Security, Privacy Issues and challenges In Cloud Computing: A Survey. Second International Conference on Information and Communication Technology for Competitive Strategies, Udaipur, India. https://doi.org/10.1145/2905055.2905253

Prieto, F., Ariaz, M. (2014). Cloud computing como ventaja competitiva en las organizaciones. Paper presented at the Twelfth LACCEI 
Latin American and Caribbean Conference for Engineering and Technology (LACCEI'2014), Guayaquil Ecuador.

Senarathna, R. (2016). Cloud computing adoption by SMEs in Australia: Deakin University. https://doi.org/10.4018/978-1-4666-9466-8. $\underline{\text { ch015 }}$

Serrano, C. (2014). Modelo para la investigacion documental. In U. d. C. F. e. I. E. y. T. G. e. I. Telemática (Ed.), Modelo para la investigacion documental.

Tomiyama, K., Kawashima, H., Kitagawa, H. (2012). A security aware stream data processing scheme on the cloud and its efficient execution methods. Paper presented at the Proceedings of the fourth international workshop on Cloud data management, Maui, Hawaii, USA. https:// doi.org/10.1145/2390021.2390033

Wong, P., He, Z., Lo, E. (2013). Parallel analytics as a service. Paper presented at the Proceedings of the 2013 ACM SIGMOD International Conference on Management of Data, New York, New York, USA. https://doi. org/10.1145/2463676.2463714

Zhang, S., Zhang, S., Chen, X., Huo, X. (2010). Cloud Computing Research and Development Trend. Paper presented at the 2010 Second International Conference on Future Networks. https://doi.org/10.1109/ICFN.2010.58

Zuberoa, M., Ocampos, J., Moral, C. d. (2017). Data center submarinos: la información de internet, guardada bajo el mar. Vodafone. 